\title{
Chronic Kidney Disease Classification in Systolic Blood Pressure Intervention Trial: Comparison Using Modification of Diet in Renal Disease and CKD-Epidemiology Collaboration Definitions
}

\author{
Michael V. Rocco ${ }^{a}$ Arlene Chapman ${ }^{b}$ Glenn M. Chertow ${ }^{c}$ Debbie Cohen ${ }^{d}$
} Jing Chen ${ }^{e}$ Jeffrey A. Cutler ${ }^{f}$ Matthew J. Diamond ${ }^{g}$ Barry I. Freedman ${ }^{a}$ Amret Hawfield $^{\mathrm{a}}$ Eric Judd $^{\mathrm{h}}$ Anthony A. Killeen ${ }^{\mathrm{j}}$ Kent Kirchner $^{\mathrm{i}}$ Cora E. Lewis $^{\mathrm{h}}$ Nicholas M. Pajewski ${ }^{\mathrm{a}}$ Barry M. Wall ${ }^{\mathrm{k}}$ Jerry Yeel for the SPRINT Research Group

\footnotetext{
a'Wake Forest School of Medicine, Winston-Salem, N.C., b University of Chicago, Chicago, III., ' Stanford University School of Medicine, Palo Alto, Calif., dPerelman School of Medicine at the University of Pennsylvania, Philadelphia, Pa., ${ }^{\text {T}}$ Tulane University School of Medicine, New Orleans, La., and ${ }^{\mathrm{f}}$ National Heart, Lung, and Blood Institute,

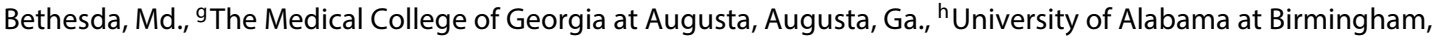
Birmingham, Ala., 'Veterans Affairs Medical Center, and University of Mississippi School of Medicine, Jackson, Miss. jUniversity of Minnesota, Minneapolis, Minn., ${ }^{k}$ University of Tennessee Center for Health Sciences and Veterans Affairs Medical Center, Memphis, Tenn., and 'Henry Ford Health System, Detroit, Mich., USA
}

\section{Key Words}

Chronic kidney disease - Modification of Diet in Renal Disease study equation - CKD-Epidemiology Collaboration equation · Hypertension · Elderly · Cardiovascular disease · Clinical trial

\footnotetext{
Abstract

Background: Interventional trials have used either the Modification of Diet in Renal Disease (MDRD) or chronic kidney disease (CKD)-Epidemiology Collaboration (CKD-EPI) equation for determination of estimated glomerular filtration rate (eGFR) to define whether participants have stages 3-5 CKD. The equation used to calculate eGFR may influence the number and characteristics of participants designated as having
}

CKD. Methods: We examined the classification of CKD at baseline using both equations in the Systolic Blood Pressure Intervention Trial (SPRINT). eGFR was calculated at baseline using fasting serum creatinine values from a central laboratory. Results: Among 9,308 participants with baseline CKD classification using the 4-variable MDRD equation specified in the SPRINT protocol, 681 (7.3\%) participants were reclassified to a less advanced CKD stage (higher eGFR) and 346 (3.7\%) were reclassified to a more advanced CKD stage (lower eGFR) when the CKD-EPI equation was used to calculate eGFR. For eGFRs $<90 \mathrm{ml} / \mathrm{min} / 1.73 \mathrm{~m}^{2}$, participants $<75$ years were more likely to be reclassified to a less advanced CKD stage; this reclassification was more likely to occur in nonblacks rather than blacks. Participants aged $\geq 75$ years were more likely to be reclassified to a more advanced than a less

\section{KARGER}

E-Mail karger@karger.com www.karger.com/ajn
(C) 2016 S. Karger AG Basel

$0250-8095 / 16 / 0442-0130 \$ 39.50 / 0$
Michael V. Rocco, MD, MSCE

Section on Nephrology

Wake Forest School of Medicine, Medical Center Boulevard

Winston-Salem, NC 27157-1053 (USA)

E-Mail mrocco@wakehealth.edu 
advanced CKD stage, regardless of baseline CKD stage. Reclassification of baseline CKD status (eGFR $<60 \mathrm{ml} /$ $\mathrm{min} / 1.73 \mathrm{~m}^{2}$ ) occurred in $3 \%$ of participants. Conclusions: Use of the MDRD equation led to a higher percentage of participants being classified as having CKD stages 3-4. Younger and non-black participants were more likely to be reclassified as not having CKD using the CKD-EPI equation.

(c) 2016 S. Karger AG, Basel

\section{Background}

The Systolic Blood Pressure Intervention Trial (SPRINT) trial is a National Institutes of Health (NIH)sponsored trial of blood pressure (BP) control in people without diabetes mellitus at increased risk of developing cardiovascular disease (CVD) [1]. Three specific subgroups were targeted for recruitment due to their increased risk of CVD, including those with prior CV events, age $\geq 75$ years and the presence of stages $3-4$ chronic kidney disease (CKD). Accurate classification of CKD will be important for analytic purposes because many of the primary and secondary outcomes will be analyzed by subgroup, including CKD status. During protocol development, the abbreviated (4-variable) Modification of Diet in Renal Disease (MDRD) study equation [2] was chosen to assess for the presence or absence of CKD. Since then, the CKD-Epidemiology Collaboration (CKD-EPI) equation [3] has been developed and deemed acceptable, if not preferred method, for estimating glomerular filtration rate (GFR). Other investigators have reported variability in the classification of CKD stages based on age and other clinical characteristics of study participants [4-11]. To our knowledge, no published analysis has determined the potential impact of applying alternatively estimated GFR (eGFR) equations on defining the presence of CKD in a randomized clinical trial. We hypothesize that some participants who would be classified as having CKD using the MDRD equation would not be found to have CKD if eGFR were calculated using the CKD-EPI equation. In this analysis, we determine the degree of reclassification of CKD stage and identify participant characteristics associated with reclassification.

\section{Methods}

Study Sample

SPRINT is a multicenter, randomized clinical trial of standard versus more intensive $\mathrm{BP}$ control in participants aged $\geq 50$ years with a heightened risk of CVD, including subgroups of prior CV events, $\geq 75$ years and/or the presence of CKD. Details of the intervention and outcomes have been previously published [1, 12]. Exclusion criteria included an eGFR $<20 \mathrm{ml} / \mathrm{min} / 1.73 \mathrm{~m}^{2}$ at baseline by the MDRD equation, diabetes mellitus, prior stroke, polycystic kidney disease and moderate- to high-grade proteinuria $(>1,000 \mathrm{mg} /$ day) or albuminuria ( $>600 \mathrm{mg} /$ day). Participants were randomized to a systolic $\mathrm{BP}$ target of either $<140 \mathrm{~mm} \mathrm{Hg}$ (standard arm) or $<120 \mathrm{~mm} \mathrm{Hg}$ (intensive arm). The primary composite end point, including adjudicated CV death, or first myocardial infarction (MI), stroke, heart failure and non-MI acute coronary syndrome was ascertained over a follow-up period of up to 6 years. The intervention was to be assessed in predefined subgroups, including participants with and without CKD (eGFR $20-<60 \mathrm{ml} /$ $\min / 1.73 \mathrm{~m}^{2}$ as calculated by the 4 -variable MDRD equation) and participants aged $<75$ or $\geq 75$ years at baseline. There were several key secondary outcomes including, in the CKD subgroup, the rate of end-stage kidney disease (ESKD) or a 50\% decline from baseline eGFR, and in the non-CKD subgroup, a $30 \%$ decrease from baseline eGFR with an end value of $<60 \mathrm{ml} / \mathrm{min} / 1.73 \mathrm{~m}^{2}$. Participant selection occurred across the United States with 102 clinical sites in the United States, including Puerto Rico. Written informed consent was obtained from all participants.

\section{Study Measurements}

Standardized questionnaires were completed during screening in order to characterize participants based on socioeconomic factors. Self-reported race/ethnicity was categorized as white, black or other and Hispanic or non-Hispanic. The Framingham Risk Score (FRS) for 10 -year CVD risk of $\geq 15 \%$ was calculated using lipid values assessed in the previous 12 months [13]. The occurrence of cardiovascular and peripheral arterial events during the previous 10 years was obtained at baseline from patient history and a review of medical records. BP was measured at baseline using a standardized protocol. A central laboratory performed all blood and urine laboratory determinations. Participants were asked to fast for $\geq 8 \mathrm{~h}$ on the day that the blood was collected.

The SPRINT Central Lab measured creatinine in serum and urine on a Roche Chemistry Analyzers (Roche Diagnostics Corporation, Indianapolis, Ind., USA) using a creatinase enzymatic method with calibration traceable to an isotope dilution mass spectrometry (IDMS) procedure. Urine albumin was measured by nephelometry using the Siemens ProSpec nephelometer and a rabbit-derived anti-human albumin antibody. The urine albumin/ creatinine ratio (UACR) was calculated using a spot urine sample obtained at the baseline visit and measured in milligrams of albumin per gram of creatinine $(\mathrm{mg} / \mathrm{g})$. Baseline eGFR in $\mathrm{ml} /$ $\mathrm{min} / 1.73 \mathrm{~m}^{2}$ was calculated by the 4 -variable MDRD equation using the serum creatinine concentration obtained at the baseline visit. CKD categories were defined using Kidney Disease Improving Global Outcomes guidelines, with the proviso that CKD classification was based on a single determination of eGFR [14].

\section{Statistical Analysis}

For both the MDRD and CKD-EPI study equations, eGFR was categorized as $\geq 90,60-89,45-59,30-44$ and $15-29 \mathrm{ml} / \mathrm{min} / 1.73 \mathrm{~m}^{2}$. Baseline characteristics of the SPRINT cohort were then compared by reclassification status based on the CKD-EPI equation (reclassified to a less advanced CKD stage (higher eGFR), not reclassified or reclassified to a more advanced CKD stage (lower eGFR) using (as appropriate) analysis of variance/Kruskal-Wallis tests for con- 
tinuous variables and chi-square tests/Fisher's exact tests for categorical variables). Based on the $5 \times 5$ cross-tabulation of eGFR categories, agreement was also assessed using kappa statistics with Fleiss-Cohen weights [15]. Of the 9,361 participants randomized, there were 53 participants in whom CKD status could not be ascertained at baseline, and these participants were excluded from these analyses. All statistical analyses were performed using SAS 9.4 (SAS Institute, Cary, N.C., USA) or the R Statistical Computing Environment.

\section{Results}

There were 9,308 SPRINT participants in whom CKD status at baseline was determined. The mean age was $67.9 \pm 9.4$ years; $65 \%$ were men. Racial and ethnic composition included approximately $30 \%$ black and $10 \%$ Hispanic. Subgroups at baseline included $28.4 \%$ with CKD $\left(\mathrm{eGFR}_{\mathrm{MDRD}}<60 \mathrm{ml} / \mathrm{min} / 1.73 \mathrm{~m}^{2}\right), 28.1 \%$ aged $\geq 75$ years and $20.1 \%$ who had prior CVD. Overall, the median (25th-75th percentile) eGFR ${ }_{\text {MDRD }}$ was $71.2 \mathrm{ml} / \mathrm{min} /$ $1.73 \mathrm{~m}^{2}$ (58.1-84.4) and the median (25th-75th percentile) UACR was $9.5 \mathrm{mg} / \mathrm{g}(5.6-21.4)$.

Compared to the original classification of $\mathrm{CKD}$ by the MDRD equation, 1,027 participants (11.0\%) were reclassified into a different CKD category using the CKD-EPI equation, including 681 participants $(7.3 \%)$ who were reclassified to a less advanced CKD stage (higher eGFR) and 346 participants $(3.7 \%)$ who were reclassified to a more advanced CKD stage (lower eGFR). Characteristics associated with a reclassification to a less advanced CKD stage, based on univariate analysis, included younger age, female sex, white race, lower FRS, higher low-density lipoprotein cholesterol level, lower high-density lipoprotein cholesterol level, higher diastolic BP, absence of prior cardiovascular history and lower urine protein or urine albumin excretion. Selected baseline characteristics of the SPRINT participants, according to reclassification status, are shown in table 1.

More than $10 \%$ of participants with a baseline MDRD eGFR of $\geq 90 \mathrm{ml} / \mathrm{min} / 1.73 \mathrm{~m}^{2}$ were reclassified to a more advanced CKD stage when eGFR was calculated using the CKD-EPI equation (fig. 1). Conversely, 8.7, 11.2 and 5.1\% of participants, respectively, with MDRD eGFRs of 60 $89,45-69$ and $30-44 \mathrm{ml} / \mathrm{min} / 1.73 \mathrm{~m}^{2}$ were reclassified to a less advanced CKD stage when the CKD-EPI equation was employed. Age has a substantial impact on CKD reclassification in our cohort. For the age category of 50-64 years, $17.9,25.6$ and $11.5 \%$ of participants, respectively, with MDRD GFRs of 60-89, 45-69 and 30-44 ml/ $\mathrm{min} / 1.73 \mathrm{~m}^{2}$ were reclassified to a less advanced stage of
CKD while the level of reclassification to a less advanced stage of CKD in the age category of $\geq 75$ years was consistently $\leq 1.5 \%$ (fig. 2). Conversely, for the age category of $\geq 75$ years, $66.3,5.6,7.6$ and $4.8 \%$ of participants, respectively, with MDRD GFRs of $\geq 90,60-89,45-69$ and 30-44 $\mathrm{ml} / \mathrm{min} / 1.73 \mathrm{~m}^{2}$ were reclassified to a more advanced stage of CKD. Reclassification to a more advanced stage of CKD in the 50-64 years or older age group rarely occurred across all CKD categories.

Reclassification status based on race and sex is depicted in figure 3. Non-black men and women had similar patterns of reclassification, with $21-23 \%$ reclassification to a lower eGFR if the MDRD calculated eGFR was $\geq 90$ $\mathrm{ml} / \mathrm{min} / 1.73 \mathrm{~m}^{2}$ and higher rates of reclassification to higher levels of eGFR than to lower levels of eGFR at MDRD eGFRs $\leq 90 \mathrm{ml} / \mathrm{min} / 1.73 \mathrm{~m}^{2}$. A similar pattern was seen for Hispanics; however, there was a higher rate of reclassification to higher levels of eGFR than in whites. Black men and women had low levels of reclassification at eGFRs of $\geq 90 \mathrm{ml} / \mathrm{min} / 1.73 \mathrm{~m}^{2}$ and were less likely to be reclassified compared to non-blacks of the same sex at lower levels of MDRD eGFRs.

There were 2,742 (29.5\%) participants who were classified as having CKD using either or both of the estimating equations for eGFR. This subgroup included 198 participants who had an MDRD eGFR $<60 \mathrm{ml} / \mathrm{min} / 1.73 \mathrm{~m}^{2}$ and a CKD-EPI eGFR $>60 \mathrm{ml} / \mathrm{min} / 1.73 \mathrm{~m}^{2}$ and 85 participants who had an MDRD eGFR $>60 \mathrm{ml} / \mathrm{min} / 1.73 \mathrm{~m}^{2}$ and a CKD-EPI eGFR $<60 \mathrm{ml} / \mathrm{min} / 1.73 \mathrm{~m}^{2}$ (table 2). Variables associated with reclassification of this subgroup were similar to that seen in the entire SPRINT cohort.

The agreement between eGFR categories using the 2 eGFR estimating equations, as determined by weighted kappa statistics (table 3), was also associated with subject characteristics, with lower levels of agreement seen in the 50-65 years age group, other races, Hispanic ethnicities and lower levels of albuminuria.

\section{Discussion}

In this large sample of non-diabetic individuals at increased risk of cardiovascular events including more than one in 4 with CKD, $7.3 \%$ of participants were reclassified to a less advanced CKD stage (higher eGFR) and 3.7\% were reclassified to a more advanced CKD stage (lower eGFR) when the CKD-EPI equation was used to calculate eGFR. Age was the most important factor in reclassification. Participants $<75$ years of age more frequently had a 
Table 1. Characteristics of SPRINT participants according to reclassification status

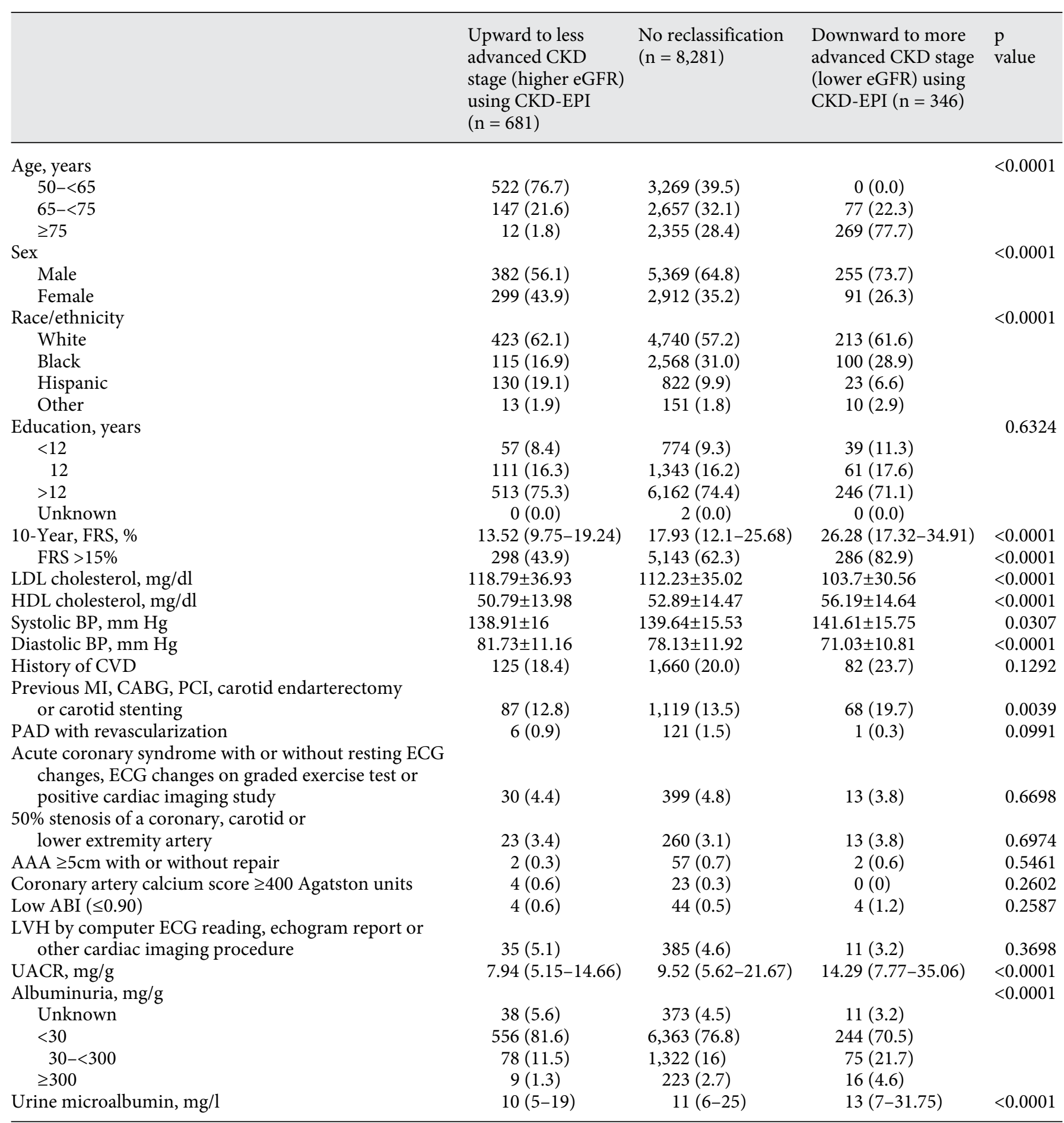

Values are $\mathrm{n}(\%)$, mean $\pm \mathrm{SD}$, or median (interquartile range).

$\mathrm{LDL}=$ Low-density lipoprotein; HDL = high-density lipoprotein; CABG = coronary artery bypass grafting; PCI = percutaneous coronary intervention; $\mathrm{PAD}=$ peripheral artery disease; $\mathrm{AAA}=$ abdominal aortic aneurysm; $\mathrm{ABI}=$ ankle brachial index; $\mathrm{LVH}=$ left ventricular hypertrophy. 
Fig. 1. Reclassification rates for SPRINT participants $(n=9,308)$ across eGFR categories by the CKD-EPI and MDRD study equations. Blue bars indicate reclassification to less advanced CKD stage (higher eGFR); yellow bars, no reclassification; red bars, downward reclassification to more advanced CKD stage (lower eGFR).

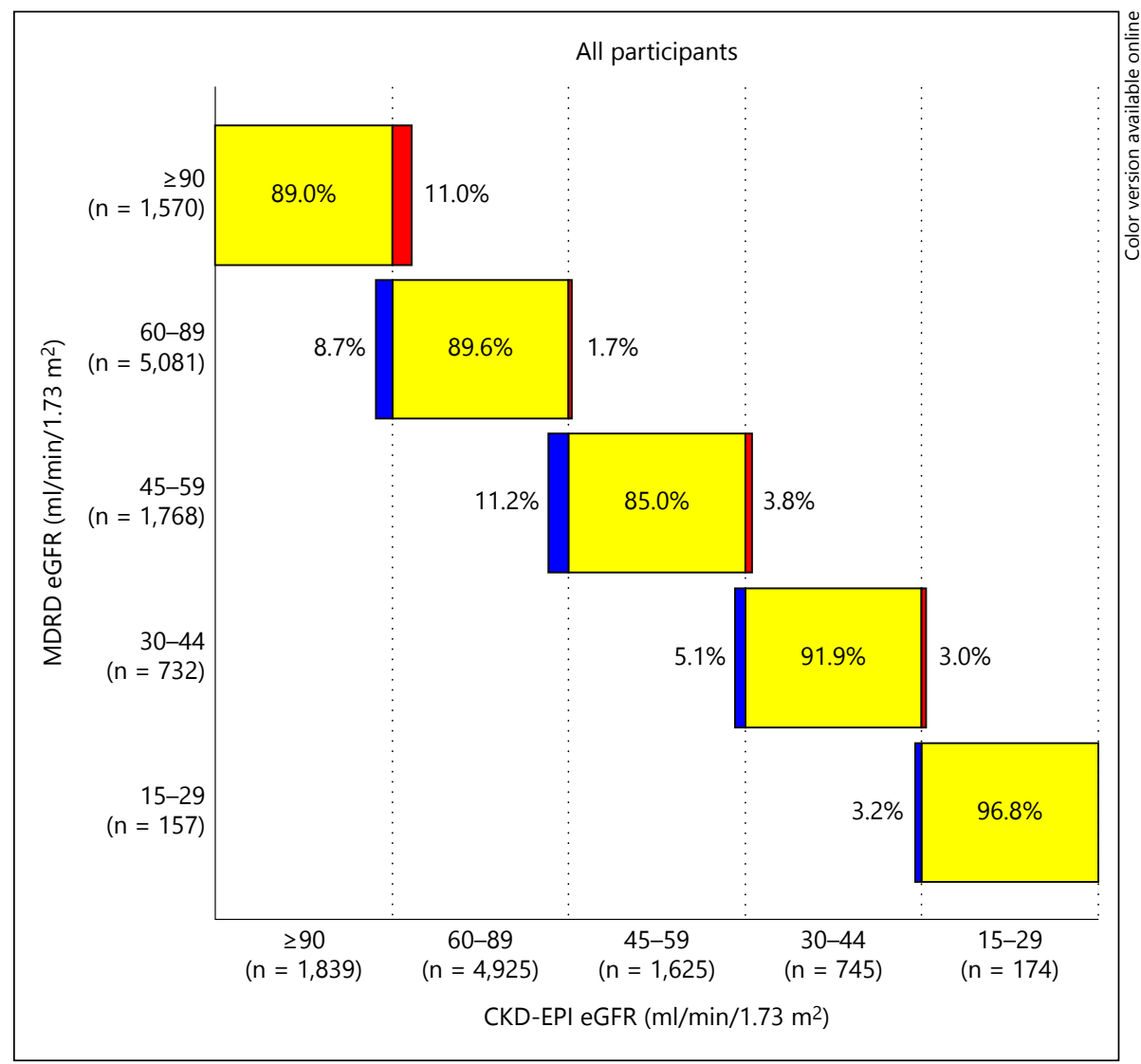

higher eGFR using the CKD-EPI equation, while participants aged $\geq 75$ years more frequently had a lower eGFR using the CKD-EPI equation. The highest agreement between eGFR equations was seen among black participants, and this group had the lowest frequency of eGFR reclassification. Reclassification resulting in a change in (dichotomous) baseline CKD status $\left(<60 \mathrm{ml} / \mathrm{min} / 1.73 \mathrm{~m}^{2}\right)$ was observed in $3 \%$ of participants.

There are several limitations to the use of either the MDRD equation or the CKD-EPI equation for the estimation of GFR. First, it is important to consider the populations studied for the validation of these 2 estimating equations. The MDRD equation was validated in 1,085 patients with an eGFR of $<60 \mathrm{ml} / \mathrm{min} / 1.73 \mathrm{~m}^{2}$ who did not have either insulin-requiring diabetes mellitus or a body weight $>160 \%$ standard body weight [2] .

The MDRD cohort was younger than the SPRINT cohort, with a mean age of $51 \pm 13$ years and only included participants with CKD. A subsequent validation study was conducted in 5,504 individuals who were younger than those in the SPRINT cohort, with a mean age of $47 \pm$ 13 years [16]. The development and validation of the
CKD-EPI equation included participants who were younger than those in the SPRINT cohort, with mean ages of $47 \pm 15$ and $50 \pm 15$ years, respectively [2]. In fact, a minority of the individuals used in either sample were $\geq 70$ years.

Second, other investigators have compared both estimating equations to other more formal measures of GFR and have found that the CKD-EPI equation has greater precision and accuracy. Earley et al. [11] performed a systematic review of studies that compared a reference GFR method with both the MDRD and CKD-EPI eGFR equations. For the 12 studies conducted in North America, Europe and Australia, the CKD-EPI equation performed better at higher GFRs (approximately $>60 \mathrm{ml} / \mathrm{min} /$ $1.73 \mathrm{~m}^{2}$ ) while the MDRD equation performed better at lower GFRs. For the 8 studies conducted in Asian or black populations, which used a modified MDRD equation to improve performance in the local population, neither equation performed as well as they did in North American or European populations. The large study $(n=5,238)$ of Murata et al. [17] observed that, compared to a gold standard ${ }^{125} \mathrm{I}$-iothalamate measured GFR, both estimat- 

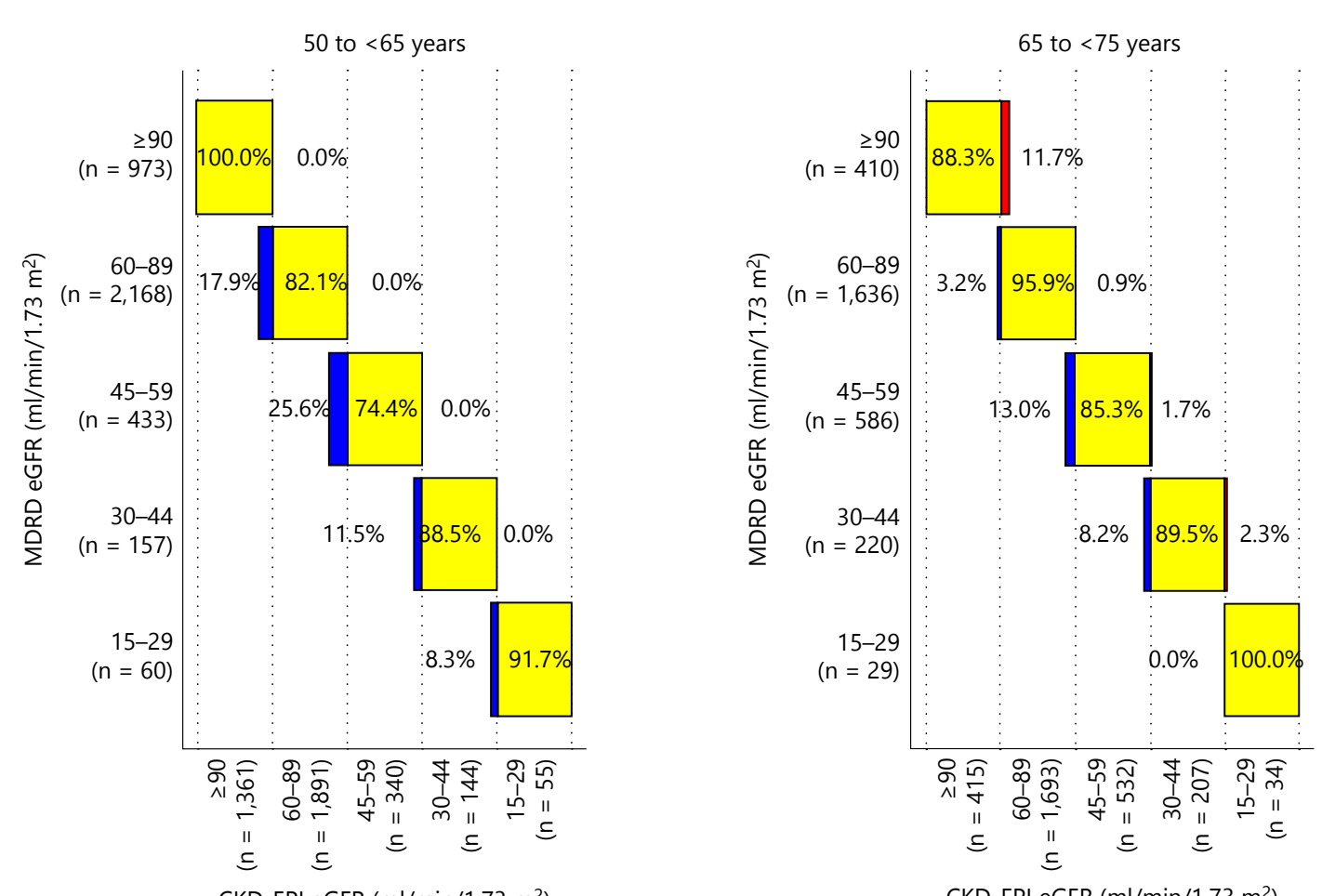

CKD-EPI eGFR (ml/min/1.73 $\left.\mathrm{m}^{2}\right)$

CKD-EPI eGFR (ml/min/1.73 $\left.\mathrm{m}^{2}\right)$

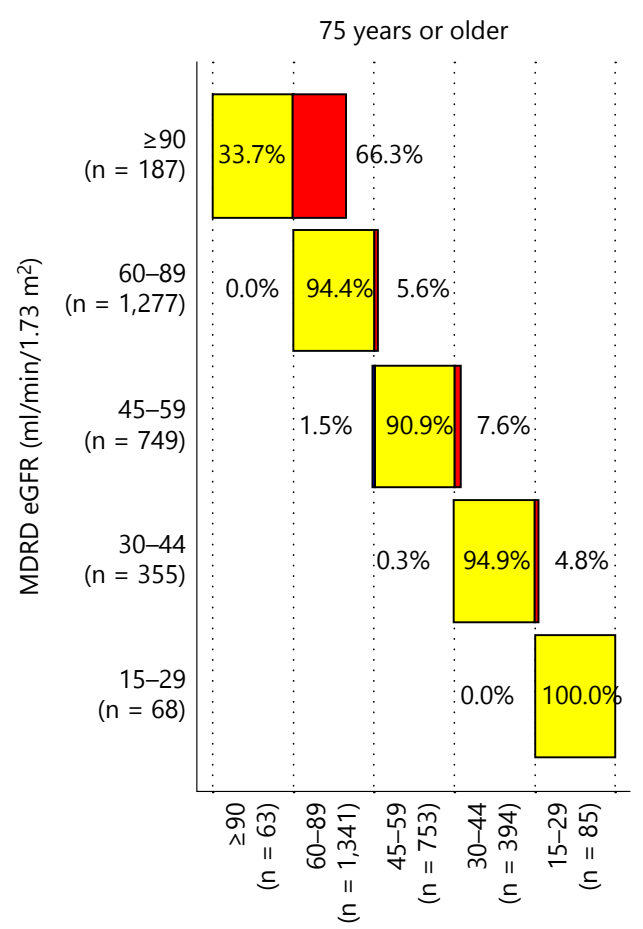

CKD-EPI eGFR $\left(\mathrm{ml} / \mathrm{min} / 1.73 \mathrm{~m}^{2}\right)$

Fig. 2. Reclassification rates for SPRINT participants by age across eGFR categories by the CKD-EPI and MDRD study equations. Blue bars indicate reclassification to less advanced CKD stage (higher eGFR); yellow bars, no reclassification; red bars, downward reclassification to more advanced CKD stage (lower eGFR). 


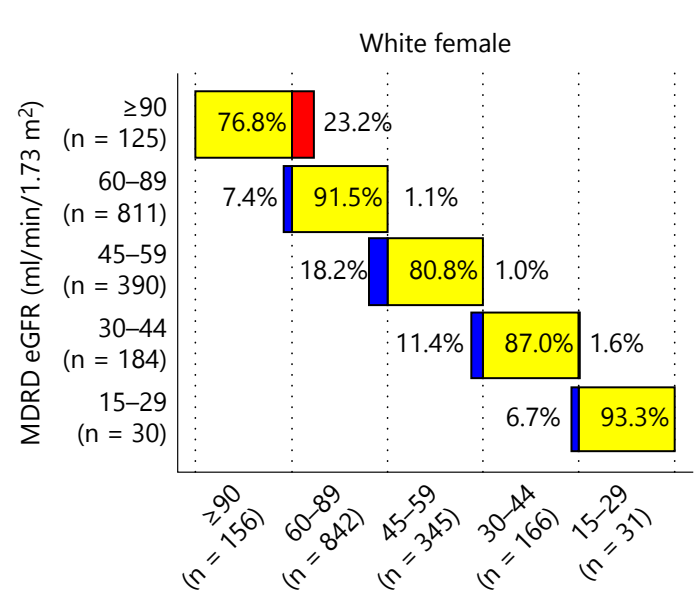

CKD-EPI eGFR (ml/min/1.73 $\left.\mathrm{m}^{2}\right)$
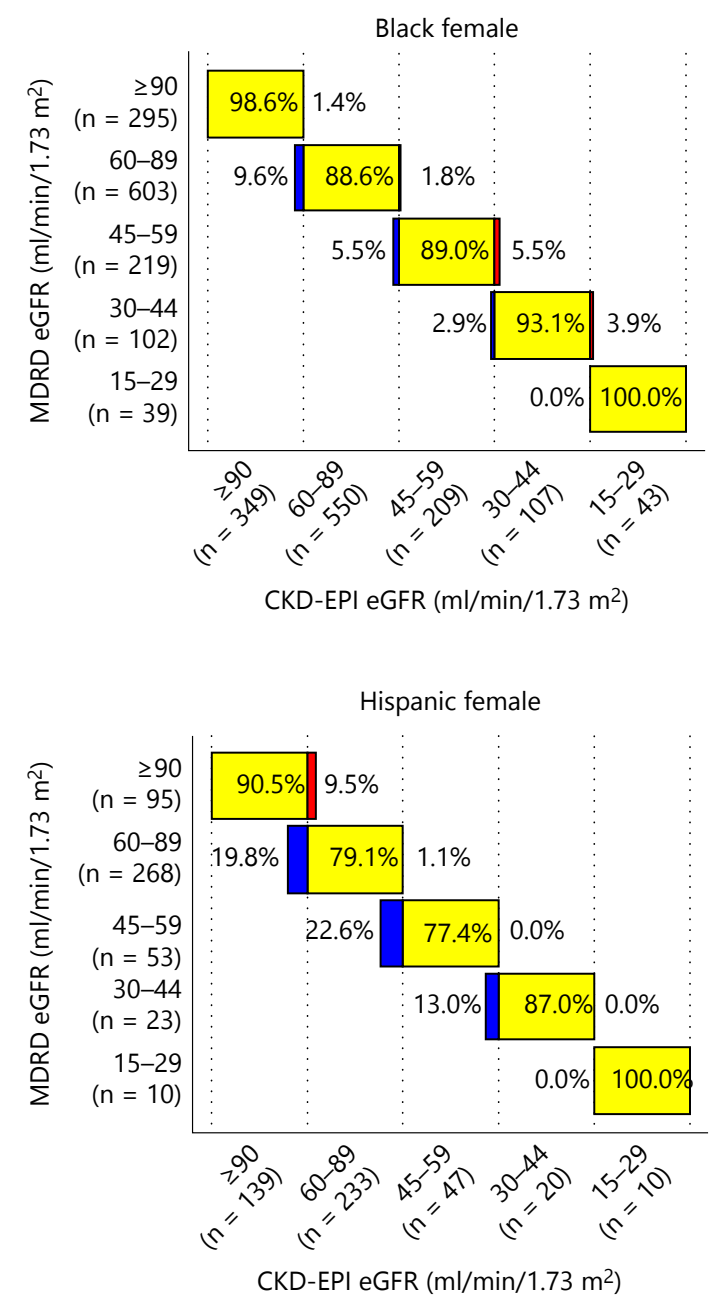
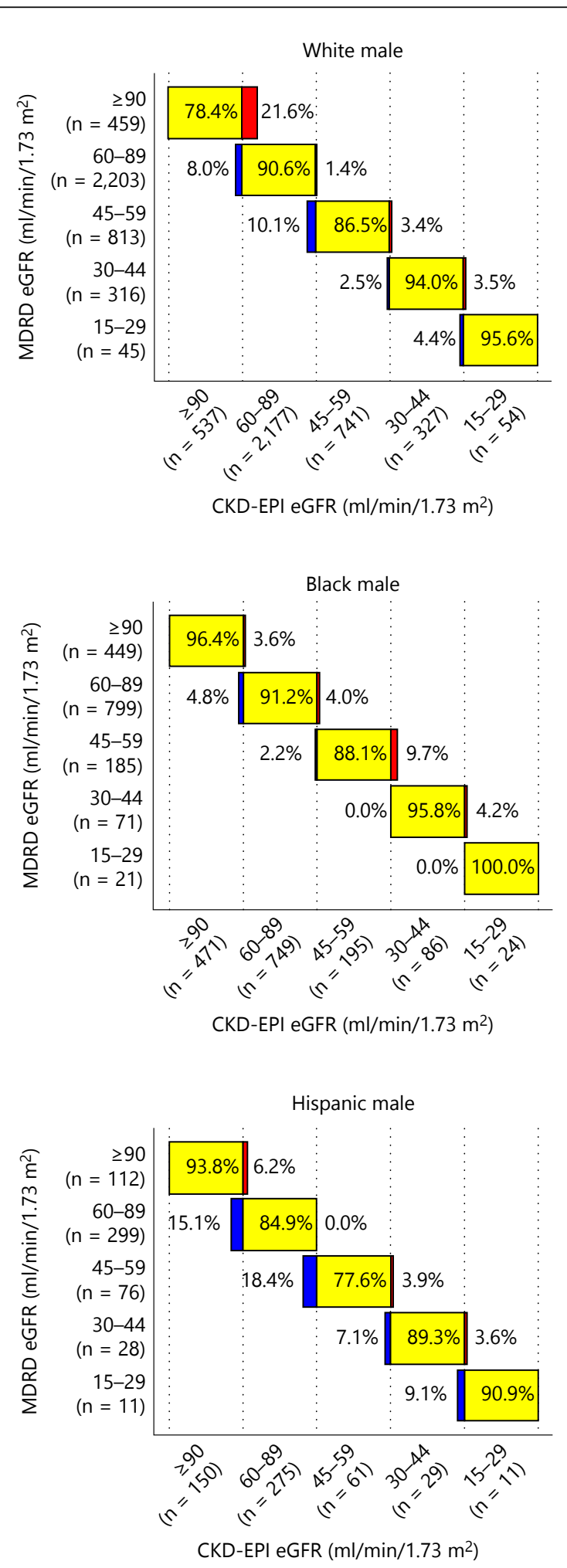

Fig. 3. Reclassification rates for SPRINT participants by race (black or white only) Hispanic ethnicity and gender across eGFR categories by the CKD-EPI and MDRD study equations. Blue bars indicate reclassification to less advanced CKD stage (higher eGFR); yellow bars, no reclassification; red bars, downward reclassification to more advanced CKD stage (lower eGFR). 
Table 2. Reclassification of SPRINT participants with eGFR $<60 \mathrm{ml} / \mathrm{min} / 1.73 \mathrm{~m}^{2}$ based on either MDRD or CKD-EPI study equations

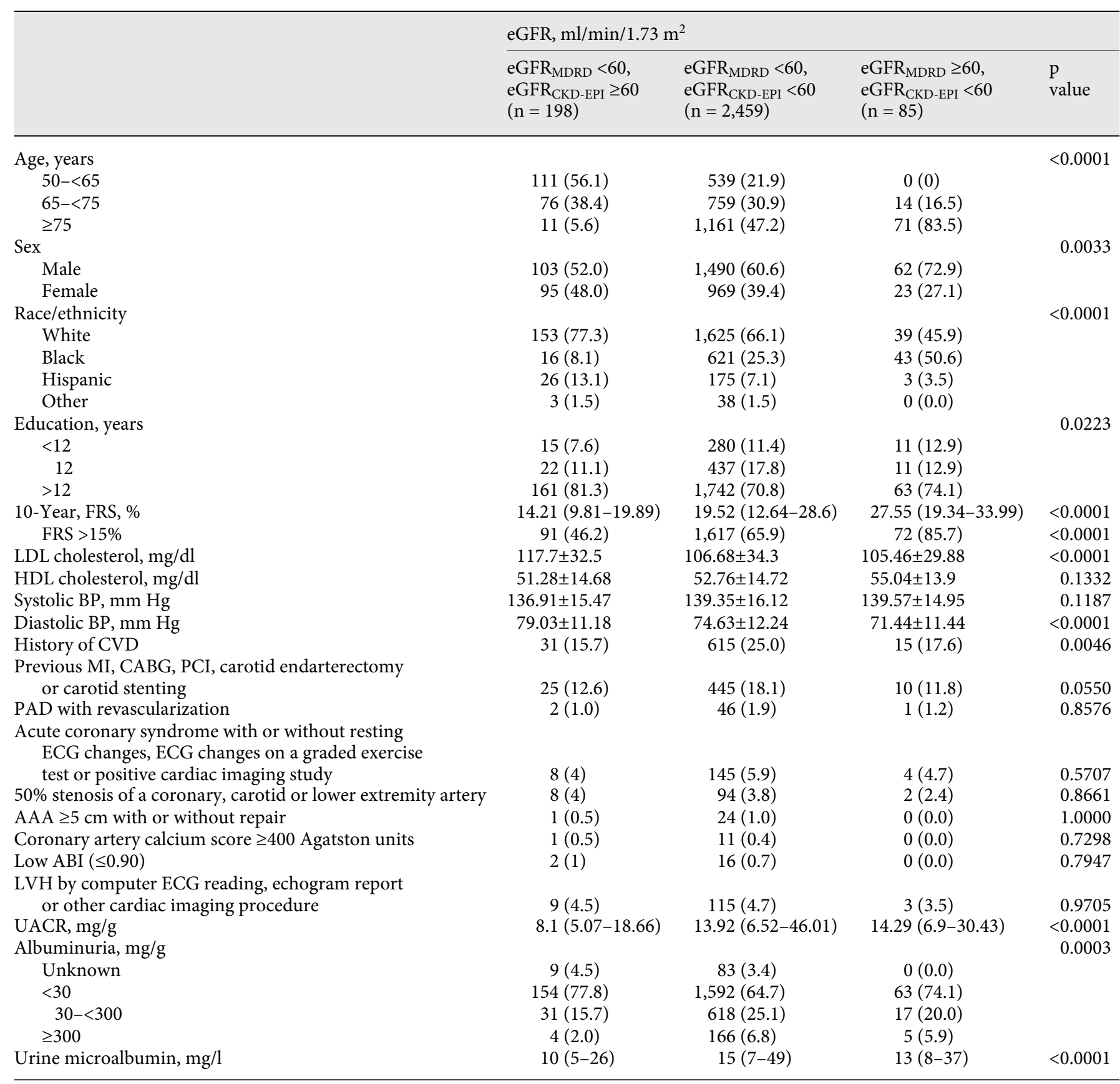

Values are $\mathrm{n}(\%)$, mean $\pm \mathrm{SD}$, or median (interquartile range). $\mathrm{LDL}=$ Low-density lipoprotein; HDL = high-density lipoprotein; CABG = coronary artery bypass grafting; $\mathrm{PCI}=$ percutaneous coronary intervention; $\mathrm{PAD}=$ peripheral artery disease; $\mathrm{AAA}=\mathrm{abdominal}$ aortic aneurysm; $\mathrm{ABI}=$ ankle brachial index; $\mathrm{LVH}=$ left ventricular hypertrophy.

ing equations overestimated GFR in individuals $>70$ years of age who had CKD ( $9 \%$ for MDRD eGFR vs. $5 \%$ for CKD-EPI) and underestimated GFR in younger healthy individuals $(-17 \%$ for MDRD eGFR vs. $-9 \%$ for CKDEPI). In a study of 392 elderly individuals who were at least 74 years of age, Kilbride et al. [5] measured GFR by the iohexol clearance method and found the that CKDEPI equation appeared less biased and was more accurate than the MDRD equation. Both equations overestimated measured GFR; however, this was only significant for the 
Table 3. Agreement between eGFR categories by the CKD-EPI and MDRD study equations

\begin{tabular}{|c|c|c|c|}
\hline & \multirow[t]{2}{*}{ Number } & \multicolumn{2}{|c|}{ Kappa statistic (95\% CI) } \\
\hline & & unweighted & weighted $^{\mathrm{a}}$ \\
\hline \multicolumn{4}{|l|}{ Age, years } \\
\hline $50-<65$ & 3,791 & $0.77(0.76-0.79)$ & $0.90(0.89-0.91)$ \\
\hline $65-<75$ & 2,881 & $0.87(0.85-0.89)$ & $0.94(0.93-0.95)$ \\
\hline$\geq 75$ & 2,636 & $0.84(0.82-0.85)$ & $0.93(0.93-0.94)$ \\
\hline \multicolumn{4}{|l|}{ Sex } \\
\hline Male & 6,006 & $0.83(0.82-0.84)$ & $0.93(0.92-0.93)$ \\
\hline Female & 3,302 & $0.82(0.80-0.84)$ & $0.93(0.93-0.94)$ \\
\hline \multicolumn{4}{|l|}{ Race/ethnicity } \\
\hline White & 5,376 & $0.81(0.79-0.82)$ & $0.92(0.91-0.93)$ \\
\hline Black & 2,783 & $0.88(0.87-0.90)$ & $0.96(0.95-0.96)$ \\
\hline Hispanic & 975 & $0.75(0.71-0.78)$ & $0.90(0.88-0.92)$ \\
\hline Other & 174 & $0.78(0.70-0.87)$ & $0.89(0.85-0.94)$ \\
\hline \multicolumn{4}{|c|}{ Race/ethnicity and sex } \\
\hline White female & 1,540 & $0.80(0.77-0.82)$ & $0.92(0.90-0.93)$ \\
\hline White male & 3,836 & $0.81(0.80-0.83)$ & $0.92(0.91-0.93)$ \\
\hline Black female & 1,258 & $0.88(0.86-0.90)$ & $0.96(0.95-0.97)$ \\
\hline Black male & 1,525 & $0.89(0.86-0.91)$ & $0.95(0.94-0.96)$ \\
\hline Hispanic female & 449 & $0.71(0.65-0.77)$ & $0.88(0.85-0.92)$ \\
\hline Hispanic male & 526 & $0.78(0.73-0.82)$ & $0.91(0.89-0.93)$ \\
\hline \multicolumn{4}{|l|}{ FRS $>15 \%$} \\
\hline No & 3,553 & $0.81(0.79-0.82)$ & $0.93(0.92-0.93)$ \\
\hline Yes & 5,727 & $0.84(0.83-0.85)$ & $0.93(0.93-0.94)$ \\
\hline \multicolumn{4}{|l|}{ History of CVD } \\
\hline No & 7,441 & $0.83(0.81-0.84)$ & $0.93(0.92-0.93)$ \\
\hline Yes & 1,867 & $0.83(0.81-0.85)$ & $0.94(0.93-0.94)$ \\
\hline \multicolumn{4}{|l|}{ Albuminuria, mg/g } \\
\hline Unknown & 422 & $0.80(0.74-0.85)$ & $0.89(0.86-0.92)$ \\
\hline$<30$ & 7,163 & $0.82(0.80-0.83)$ & $0.91(0.91-0.92)$ \\
\hline $30-<300$ & 1,475 & $0.86(0.83-0.88)$ & $0.95(0.95-0.96)$ \\
\hline$\geq 300$ & 248 & $0.87(0.82-0.92)$ & $0.96(0.95-0.98)$ \\
\hline
\end{tabular}

MDRD equation when the measured GFR was $\geq 60 \mathrm{ml} /$ $\mathrm{min} / 1.73 \mathrm{~m}^{2}$. This effect was most pronounced for individuals aged $\geq 80$ years.

Third, large retrospective meta-analyses have also demonstrated that use of the CKD-EPI equation improved risk stratification compared to the MDRD equation. Matsushita et al. [10] examined 1,010,988 participants in the Alberta, Canada, laboratory registry in whom outcome data were available for all-cause mortality, acute MI, ESKD and a doubling of the serum creatinine concentration. When the CKD-EPI equation was used instead of the MDRD equation, $22.6 \%$ of all participants (mean age $43.1 \pm 11.8$ years) were reclassified to a less advanced CKD stage. Conversely, the use of the CKD-EPI equation reclassified $1.2 \%$ of all par- ticipants (mean age $81.1 \pm 6.8$ years) to a more advanced CKD stage. The reported prevalence of CKD stages 3 and 4 changed from 9.2 to $7.3 \%$. Similarly, data from the Third National Health and Nutrition Examination Survey showed that use of the CKD-EPI equation resulted in $26.9 \%$ of the population being reclassified to a less advanced CKD stage and $2.2 \%$ being reclassified to a more advanced CKD stage. Characteristics associated with being reclassified to a less advanced CKD stage included younger age, female sex and lower prevalence of CVD and diabetes mellitus [7]. Finally, a meta-analysis of 45 studies comprising 1,130,472 adults, including those from the general population, those with CKD and those at risk for CKD determined that $24.4 \%$ of participants from the general population cohort were 
reclassified to a less advanced $\mathrm{CKD}$ stage when the MDRD eGFR equation was replaced by the CKD-EPI equation, while $0.6 \%$ were reclassified to a more advanced CKD stage. This reclassification resulted in a decrease in the percentage of participants having stages 3-4 CKD from 8.7 to $6.3 \%$. In the eGFR category of $45-59 \mathrm{ml} / \mathrm{min} / 1.73 \mathrm{~m}^{2}$, as determined by the MDRD equation, $34.7 \%$ of participants were reclassified to less advanced CKD by the CKD-EPI equation and these reclassified participants had lower levels of events (allcause and cardiovascular mortality or ESKD) compared to participants who were not reclassified [9]. In all 3 studies, reclassification of $\mathrm{CKD}$ with the CKD-EPI equation improved risk stratification; in the latter study, this improvement was noted in multiple subgroups including elderly ( $\geq 65$ years) versus non-elderly, sex, race and the presence or absence of hypertension and diabetes mellitus.

The strengths of this study include a large, racially diverse population that includes $30 \%$ blacks and $10 \%$ Hispanics as well as measurement of serum creatinine in a central laboratory using a method that has been standardized using a standardized and validated IDMS method. The findings from this study are limited by the lack of a measured GFR as a comparator. Results should be taken in the context of the study population in SPRINT; there may be more or less reclassification in other populations such as in patients with diabetes mellitus.

In conclusion, use of the MDRD equation led to a higher proportion of SPRINT participants being classified as having CKD stages 3-4. Participants who were younger or non-black were more likely to be reclassified as not having $\mathrm{CKD}$ when considering the CKD-EPI equation as the criterion standard.

\section{Acknowledgments}

The investigators would like to acknowledge the SPRINT participants without whom this trial would not have been possible. We also acknowledge the study staff in the 102 clinics in the SPRINT trial.

The SPRINT is funded with federal funds from the NIH, including the National Heart, Lung, and Blood Institute (NHLBI), the National Institute of Diabetes and Digestive and Kidney Diseases, the National Institute on Aging and the National Institute of Neurological Disorders and Stroke under contract numbers HHSN 268200900040C, HHSN268200900046C, HHSN268200900047C, HHSN268200900048C, HHSN268200900049C, and inter-agency agreement number A-HL-13-002-001. It was also supported in part with resources and use of facilities through the Department of Vet- erans Affairs. The SPRINT investigators acknowledge the contribution of study medications (azilsartan and azilsartan combined with chlorthalidone) from Takeda Pharmaceuticals International, Inc. All components of the SPRINT study protocol were designed and implemented by the investigators. The investigative team collected, analyzed and interpreted the data. All aspects of manuscript writing and revision were carried out by the coauthors. The content is solely the responsibility of the authors and does not necessarily represent the official views of the NIH, the US Department of Veterans Affairs or the United States Government. For a full list of contributors to SPRINT, see the online supplementary acknowledgement list (see www.karger.com/doi/10.1159/000448722): ClinicalTrials.gov identifier: NCT01206062.

We also acknowledge the support from the following CTSAs funded by NCATS: CWRU: UL1TR000439, OSU: UL1RR025755, U Penn:UL1RR024134 and UL1TR000003, Boston:UL1RR025771, Stanford: UL1TR000093, Tufts: UL1RR025752, UL1TR000073 and UL1TR001064, University of Illinois: UL1TR000050, University of Pittsburgh: UL1TR000005, UT Southwestern: 9U54T R000017-06, University of Utah: UL1TR000105-05, Vanderbilt University: UL1 TR000445, George Washington University: UL1TR000075, University of CA, Davis: UL1 TR000002, University of Florida: UL1 TR000064, University of Michigan: UL1TR000433, Tulane University: P30GM103337 COBRE Award NIGMS.

The authors also acknowledge Dr. Jeffrey A. Cutler's role in the initial consideration of this trial concept during 2003-2007, which led to the subsequent sponsorship of SPRINT by NHLBI.

\section{Support and Financial Disclosure Declaration}

A.C. was a consultant for Kadmon, Otsuka. C.E.L. had research funding (paid to the institution) from Novo Nordisk. All other authors have no relevant disclosures.

References

1 Ambrosius WT, Sink KM, Foy CG, Berlowitz DR, Cheung AK, Cushman WC, Fine LJ, Goff DC Jr, Johnson KC, Killeen AA, Lewis CE, Oparil S, Reboussin DM, Rocco MV, Snyder JK, Williamson JD, Wright JT Jr, Whelton PK: The design and rationale of a multicenter clinical trial comparing two strategies for control of systolic blood pressure: the systolic blood pressure intervention trial (SPRINT). Clin Trials 2014;11:532-546.

2 Levey AS, Bosch JP, Lewis JB, Greene T, Rogers N, Roth D: A more accurate method to estimate glomerular filtration rate from serum creatinine: a new prediction equation. Modification of diet in renal disease study group. Ann Intern Med 1999;130: 461-470.

3 Levey AS, Stevens LA, Schmid CH, Zhang YL, Castro AF 3rd, Feldman HI, Kusek JW, Eggers P, Van Lente F, Greene T, Coresh J: A new equation to estimate glomerular filtration rate. Ann Intern Med 2009;150:604612. 
4 Flamant M, Haymann JP, Vidal-Petiot E, Letavernier E, Clerici C, Boffa JJ, Vrtovsnik F: GFR estimation using the Cockcroft-Gault, MDRD study, and CKD-EPI equations in the elderly. Am J Kidney Dis 2012;60:847849.

5 Kilbride HS, Stevens PE, Eaglestone G, Knight S, Carter JL, Delaney MP, Farmer CK, Irving J, O'Riordan SE, Dalton RN, Lamb EJ: Accuracy of the MDRD (modification of diet in renal disease) study and CKD-EPI (CKD epidemiology collaboration) equations for estimation of GFR in the elderly. Am J Kidney Dis 2013;61:57-66.

6 Lewis JR, Lim W, Dhaliwal SS, Zhu K, Lim EM, Thompson PL, Prince RL: Estimated glomerular filtration rate as an independent predictor of atherosclerotic vascular disease in older women. BMC Nephrol 2012;13:58.

7 Shafi T, Matsushita K, Selvin E, Sang Y, Astor $\mathrm{BC}$, Inker LA, Coresh J: Comparing the association of GFR estimated by the CKD-EPI and MDRD study equations and mortality: the third national health and nutrition examination survey (NHANES III). BMC Nephrol 2012;13:42.

8 Shafi T, Parekh RS, Jaar BG, Plantinga LC, Oberai PC, Eckfeldt JH, Levey AS, Powe NR,
Coresh J: Serum $\beta$-trace protein and risk of mortality in incident hemodialysis patients. Clin J Am Soc Nephrol 2012;7:1435-1445.

9 Matsushita K, Mahmoodi BK, Woodward M, Emberson JR, Jafar TH, Jee SH, Polkinghorne KR, Shankar A, Smith DH, Tonelli M, Warnock DG, Wen CP, Coresh J, Gansevoort RT, Hemmelgarn BR, Levey AS: Comparison of risk prediction using the CKD-EPI equation and the MDRD study equation for estimated glomerular filtration rate. JAMA 2012;307: 1941-1951.

10 Matsushita K, Tonelli M, Lloyd A, Levey AS, Coresh J, Hemmelgarn BR: Clinical risk implications of the CKD epidemiology collaboration (CKD-EPI) equation compared with the modification of diet in renal disease (MDRD) study equation for estimated GFR. Am J Kidney Dis 2012;60:241-249.

11 Earley A, Miskulin D, Lamb EJ, Levey AS, Uhlig K: Estimating equations for glomerular filtration rate in the era of creatinine standardization: a systematic review. Ann Intern Med 2012;156:785-795; W270-W278.

12 Wright JT Jr, Williamson JD, Whelton PK, Snyder JK, Sink KM, Rocco MV, Reboussin DM, Rahman M, Oparil S, Lewis CE, Kimmel PL, Johnson KC, Goff DC Jr, Fine LJ, Cutler
JA, Cushman WC, Cheung AK, Ambrosius WT: A randomized trial of intensive versus standard blood-pressure control. N Engl J Med 2015;373:2103-2116.

13 D'Agostino RB Sr, Vasan RS, Pencina MJ, et al: General cardiovascular risk profile for use in primary care: the Framingham heart study. Circulation 2008;117:743-753.

14 Kidney Disease: Improving Global Outcomes (KDIGO) CKD Work Group: KDIGO 2012 clinical practice guideline for the evaluation and management of chronic kidney disease. Kidney Inter Suppl 2013;3:1-150.

15 Fleiss JL, Cohen J, Everitt BS: Large sample standard errors of kappa and weighted kappa. Psychol Bull 1969;72:323-327.

16 Stevens LA, Coresh J, Feldman HI, Greene T, Lash JP, Nelson RG, Rahman M, Deysher AE, Zhang YL, Schmid CH, Levey AS: Evaluation of the modification of diet in renal disease study equation in a large diverse population. J Am Soc Nephrol 2007;18:2749-2757.

17 Murata K, Baumann NA, Saenger AK, Larson TS, Rule AD, Lieske JC: Relative performance of the MDRD and CKD-EPI equations for estimating glomerular filtration rate among patients with varied clinical presentations. Clin J Am Soc Nephrol 2011;6:1963-1972. 